\title{
Automatic Mixing of Bio-Samples Using Micro-Channel and Centrifugation
}

\author{
Liang Yuan, Yuan F. Zheng, Fellow, IEEE, Weidong Chen, and Martin Caffrey
}

\begin{abstract}
Automatic mixing of bio-samples using microchannel and centrifugation is considered. Existing methods for mixing bio-samples for life science applications use micro-well and micro-stirrer which are ineffective when used with highly viscous materials at the microliter or nanoliter level. Our method mixes viscous bio-samples in micro-capsules using micro-channel and centrifugation which minimizes contact with mixing tools. To introduce the method, firstly the design of the micro-capsule along with the micro-channel is presented. Secondly, a hydrodynamic model describing the flow of viscous materials in the micro-channel is presented, and the average sample velocity and the traveling time through the microchannel are analyzed. Thirdly, the relationship between centrifugation speed and time is given to achieve effective and efficient control of the flow. Finally, experimental and theoretical results are compared.
\end{abstract}

\section{INTRODUCTION}

$\mathrm{I}_{\mathrm{N}}^{\mathrm{N}}$ NTEREST in micro-mixing is growing due to developments in life sciences, analytical chemistry, and food engineering. In the life sciences, one of the applications of micro-mixing is in membrane protein crystallization. Here, the so-called in meso technique requires that a highly viscous lipid (monoolein, for example) is mixed with a protein solution (bacteriorhodopsin, for example). The resultant mesophase is then dispensed into an array of wells as part of the crystallization setup [1-3]. The formed crystal can be used to determine the 3D structure of the protein [4-6; see also http://www.mpdb.ul.ie] using X-ray crystallography.

Even though a robotic system for automating the delivery of viscous bio-samples into arrays of wells (Fig. 1) has been built [7-11], the mixing of the lipid and protein is done by hand using two micro-syringes [1]. This manual process is tedious and time-consuming and must be done one sample at a time. In its current form this aspect of the method does not lend itself to automation for high-throughput

Liang Yuan is with the Department of Electrical and Computer Engineering, The Ohio State University, Columbus, OH, 43210, USA and also with the Department of Automation, Shanghai Jiao Tong University, Shanghai, 200240, China (e-mail: yuanl@ece.osu.edu).

Yuan F. Zheng (corresponding author) is with the Department of Electrical and Computer Engineering, The Ohio State University, Columbus, OH, 43210, USA and also with Shanghai Jiao Tong University, Shanghai 200240, China (e-mail: zheng@ece.osu.edu).

Weidong Chen is with the Department of Automation, Shanghai Jiao Tong University, Shanghai, 200240, China (e-mail: wdchen@sjtu.edu.cn).

Martin Caffrey is with the Department of Chemical and Environmental Sciences and Materials and Surface Science Institute, University of Limerick, Limerick, Ireland (e-mail: martin.caffrey@ul.ie). applications. Another important potential application for this type of material is in membrane protein microarrays [12]. These might be used, for example, in sensor development for medical, pharmaceutical and security applications. A proof of principle has been established [13-14]. To create such microarrays, mixing is a key step which practically cannot be done manually since there are so many wells in the microarray, the volume involved is so small (from picoliters to nanoliters), and the bio-samples are highly viscous.

At present, some micro-stirrers (agitator) [15-16] are used to mix bio-samples of low viscosity. Unfortunately, microstirrers are expensive; they are not suitable for highthroughput work and are ineffective for mixing highly viscous bio-samples because the bio-samples can easily stick to the mechanical parts of the agitators.

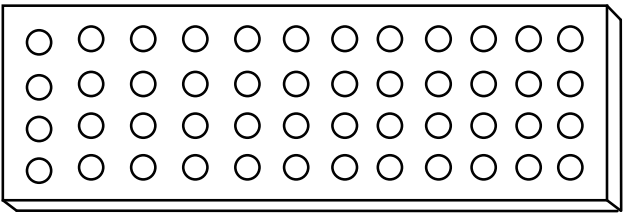

Fig. 1. A plate of passive microarray with 48 wells (containers).

Besides micro-stirrers, there are a number of different methods which provide liquid mixing on a micro-scale. These include micro-well shaking [16-17] and mechanicalchannel mixing which employs T-shape [18], Y-shape [19] and slit-type inter-digital homogenization, respectively [20]. The mechanical-channel mixing method is used for continuous mixing and flow of low viscous liquids which are pumped through the channel by a driver. However, the system is not suitable for mixing highly viscous bio-samples at the micro or nanoliter scale. Micro-well shaking on the other hand avoids the use of mechanical contact by shaking to promote mixing. Still the materials (liquids) which can be mixed are of low viscosity [21]. For highly viscous $(>3000 \mathrm{cP})$ and lower volume bio-samples $(<10 \mu \mathrm{L})$ such as lipid dispersions, the method is ineffective. Consequently, mixing bio-samples of high viscosity in small volumes represents a new challenge.

In this paper, we present an approach for the mixing of small volumes of highly viscous biomaterials in highthroughput fashion. The method makes use of a twocompartment micro-capsule with a micro-channel through which the materials are passed and mixed by centrifugation. The micro-capsules serve as a container to accommodate bio-samples for promoting and observing reactions, while the 
channels are for mixing the bio-samples. By using the centrifugal forces, the bio-samples can flow back and forth through the micro-channel to achieve mixing without contact with any moving parts. The combination of these solutions enables effective mixing of highly viscous bio-samples in small volume with minimal loss.

The most challenging issue of this new mixing mechanism is to control the flow of viscous bio-samples through the micro-channel by centrifugation, which has never been seriously addressed in both engineering and biology communities. Particularly one has to understand how viscous materials flow through the micro-channel when driven by centrifugal force. Only when this issue is clearly resolved, one can determine how fast and how long the centrifugal system should spin to generate the required centrifugal force, and consequently to achieve effective and efficient mixing of viscous bio-samples.

The paper is organized as follow. The structure of the micro-capsule is introduced in Section II. The hydrodynamic model of the flow of viscous materials in micro-channel is described in Section III. In Section IV, the control of the flow of viscous bio-samples in the micro-channel is described. Here, the relationship between the traveled volume and the centrifugal speed as well as that between the traveled volume and the required time are reported. Experimental results are presented in Section V in support of the theoretical results. Conclusions are presented in Section VI.

\section{Design OF THE Micro-CAPSUle BASED ON A Micro- CHANNEL}

The micro-capsule has two functions. Firstly, it houses the bio-samples in a chamber that can be interrogated, by spectroscopic means for example. Secondly, it provides a means whereby the contents of the capsule can be mixed in a simple and programmable way.

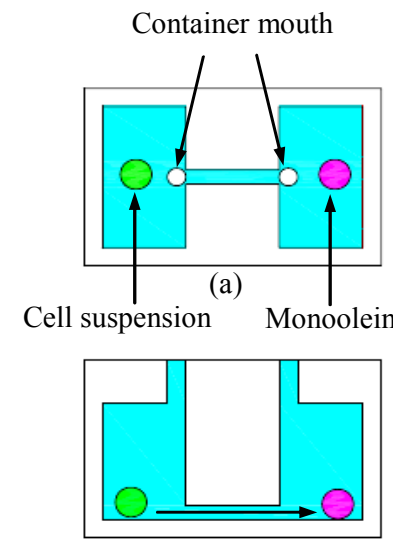

(b)

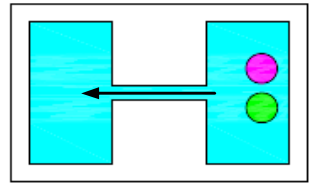

(c)

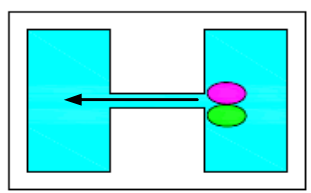

(d)
Fig. 2. Micro-capsule for mixing two bio-samples: (a), (c) and (d) are the top view, and (b) is the side view. Note that a micro-channel connecting the two chambers and each chamber has a mouth to serve as the access port.
To accommodate the above two functions, we propose the basic structure of the micro-capsule to be as shown in Fig. 2. The micro-capsule has two micro-chambers and a microchannel connecting them. The chambers are for holding the bio-samples while the micro-channel is for mixing using the channel-mixing concept. That is, homogenization is achieved when the two materials to be mixed flow through the micro-channel together as shown in Fig. 2(d). Each chamber has an access port for loading and unloading samples. The ports also allow for venting and free movement of material through the channel between chambers.

When determining the dimensions of the micro-capsule, we take the following factors into consideration. First, it should be compatible with plates which are currently used in life science and drug development applications, as shown in Fig. 1. Ideally, the footprint is identical to that of the standard microarray plate such that standard facilities for storing, shipping, and handling the plates can still be utilized. Secondly, the length and diameter of the micro-channel should be adequate for generating effective mixing. Based on these considerations, we built the micro-capsule with the dimensions shown in Fig. 3.

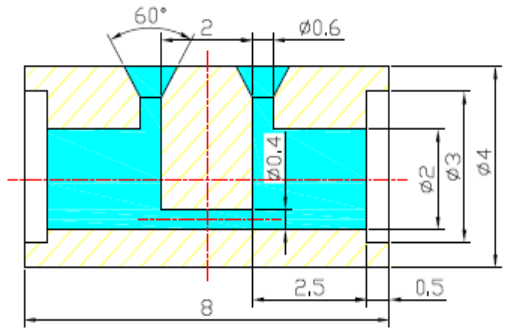

(a)

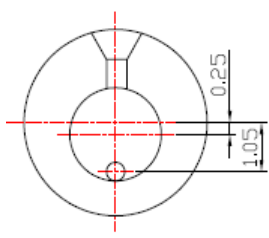

(b)
Fig. 3. The dimension of the micro-capsule: (a) front view, and (b) side view where the units are in $\mathrm{mm}$.

The micro-channel should be long and thin enough to guarantee effective mixing since the contact areas between the two materials to be mixed increase over the distance of the micro-channel [22]. In general, the longer the mixingchannel, the better mixing can be achieved. The trade-off is the increased viscous force caused by the larger area of the channel wall. Based on the volumes of the bio-samples which we have targeted $(5 \mu \mathrm{L}$ each for a total of $10 \mu \mathrm{L})$, we have chosen to make the micro-channel $2 \mathrm{~mm}$ long with a diameter of $0.4 \mathrm{~mm}$. Interestingly, these dimensions are similar to those used in the T-mixer described by Bothe et al. [22]. The other dimensions of the micro-capsule, plus that of the micro-channel, are shown in Fig. 3. Note that the diameter of the port is purposely small to minimize loss of volatiles from the sample.

\section{HydrodynAmic MOdel OF THE MiCRO-CHANNEL FLOW}

Micro-channel mixing has been an active area of study in recent years due to emerging applications in analytical chemistry and life sciences [22]. Mixing highly viscous bio- 
materials however is still an understudied topic. The mechanism we propose is to let two or more bio-samples travel though the micro-channel together such that mixing can be achieved. Primarily, we need to understand how long it takes for the viscous bio-sample to flow through the microchannel under the effect of certain centrifugal forces. To do so, we need to develop a mathematical model to describe the hydrodynamic behavior of the flow of viscous materials. Once the model becomes available, the relationship between the flow time, the viscosity and the centrifugal force can be obtained. Subsequently, an efficient high-throughput system can be developed.

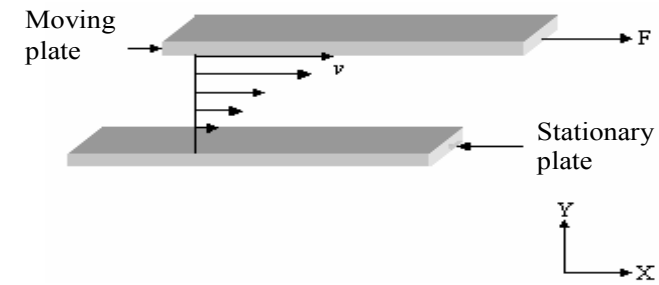

Fig. 4. Two parallel plates move with relative velocity $v$ when the space between the two is filled with viscous material.

To develop the model, a brief description of viscosity is appropriate. From [23], viscosity can be understood from a viscous material sandwiched between two flat plates separated by a distance, $y$, as shown in Fig. 4 . When the upper plate moves horizontally with a velocity $v$, and the bottom plate remains fixed, the velocity of the material decreases linearly from $v$ at the upper plate to 0 at the lower stationary plate. To create such a velocity gradient, a shear force $F$ must be applied to the upper plate, which is proportional to the coefficient of viscosity $\mu$, the velocity gradient (or shear rate) of the material $d v / d y$, and the area of the plate $A$ :

$$
F=\mu A \frac{d v}{d y} .
$$

The model of the viscous material flowing through the micro-channel for the purpose of mixing can be built based on the viscosity model of (1). Assume a micro-cylinder of length $l$ that can be as long as the micro-channel and radius $\delta$ within the micro-channel (see Fig. 5). The axis of the microcylinder is coincident with the axis of the channel. The centrifugal force acting on the bio-samples in the microcylinder (Fig. 5) can be described as follows:

$$
F_{c}=m \omega^{2} R_{c}
$$

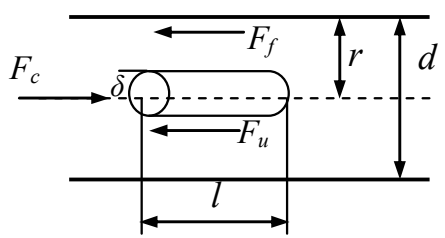

Fig. 5. The hydrodynamic model of the flow of viscous biosample in the micro-channel. where

$m$ : mass of the bio-sample in the micro-cylinder;

$\omega$ : angular velocity of the centrifuge;

$R_{c}$ : the distance between the center of rotation and the micro-cylinder.

According to (1), the shear force $F_{u}$, due to viscosity, is:

$$
F_{u}=\mu A \frac{d v}{d \delta} .
$$

There also exists frictional force between the surface of the channel and the viscous material due to the roughness of the surface. The frictional force $F_{f}$ based on Bernoulli's principle is [24]:

where $F_{f}=\pi \rho f l \bar{v}^{2} r / 4$

$\rho$ : density of the material;

$\bar{v}:$ average velocity of the material;

$f$ : frictional factor which is equal to $f=\frac{112 \mu}{\bar{v} r \rho}$.

Thus when the material flows, the summation of the frictional force $F_{f}$ and the shear force $F_{u}$ must equal the centrifugation force $F_{c}$ to guarantee a constant velocity:

$$
F_{c}=F_{u}+F_{f} .
$$

From (3) and (5), we can obtain the velocity along the radius:

$$
d v=-\frac{F_{c}-F_{f}}{2 \pi l \mu} \cdot \frac{1}{\delta} \cdot d \delta .
$$

Based on the boundary condition that $v=0$ when $\delta=r$, we can integrate equation (6):

$$
v=\frac{F_{c}-F_{f}}{2 \pi l \mu} \cdot(\ln r-\ln \delta) .
$$

Equation (7) shows that material velocity in the microchannel follows logarithmic distribution according to the micro-channel radius $r$. The maximum velocity is on the axis and the minimal velocity occurs on the micro-channel wall when $\delta=r$.

In Fig. 5, we take a small circular area of $d \delta$ at a radial position $\delta: d B=2 \pi \delta d \delta$. The flux through the circular area is:

$$
d q=v d B=v \cdot 2 \pi \delta d \delta .
$$

We substitute $v$ in equation (8) by (7):

$$
d q=\frac{F_{c}-F_{f}}{2 \pi l \mu}(\ln r-\ln \delta) \cdot 2 \pi \delta d \delta .
$$

Integrating (9), we obtain:

$$
q=\frac{F_{c}-F_{f}}{4 l \mu} r^{2} .
$$

Thus, the average velocity in the micro-channel can be expressed as: 


$$
\bar{v}=\frac{q}{\pi r^{2}}=\frac{F_{c}-F_{f}}{4 \pi l \mu} .
$$

Making use of equation (4), equation (11) becomes:

$$
\bar{v}=\frac{F_{c}}{32 \pi l \mu} .
$$

From equation (12), it is apparent that the average fluid velocity is directly proportional to the centrifugal force and inversely proportional to the channel length and the liquid viscosity.

\section{Control of the Flow of Viscous Bio-SAMPleS}

There is an important issue to resolve for the case of micro-channel mixing of viscous bio-samples, i.e., how large and how long a centrifugal force should be exerted to the bio-samples such that it can completely flow through the micro-channel? These two parameters will determine how fast and how long the centrifuge spins, i.e., the control of the flow. If the speed is too slow and the duration is too short, the bio-samples may not be able to complete passage through the micro-channel. These issues will now be discussed.

Based on equations (2) and (12), we can obtain the average velocity of flow as:

$$
\bar{v}=\frac{m \omega^{2} R_{c}}{32 \pi l \mu}=\frac{\rho \pi r^{2} l\left(\frac{2 \pi}{60}\right)^{2} n^{2} R_{c}}{32 \pi l \mu}=\frac{3.42 \times 10^{-4} \rho r^{2} n^{2} R_{c}}{\mu}
$$

where:

$n$ : speed of spin of the centrifuge (rpm).

The time $\left(t_{h}\right)$ for passage through the micro-channel can be expressed as

$$
t_{h}=\frac{l}{\bar{v}} \cdot\left(\frac{V}{V_{c}}+1\right)=\left(\frac{V}{V_{c}}+1\right) \cdot \frac{\mu l}{3.42 \times 10^{-4} \rho r^{2} n^{2} R_{c}}
$$

where

$V:$ volume of sample injected into the chamber $\left(\mathrm{m}^{3}\right)$;

$V_{c}$ : volume of the micro-channel which is equal to $\pi r^{2} l\left(\mathrm{~m}^{3}\right)$.

For the micro-channel as shown in Fig. 3, one can calculate the corresponding passage-times $\left(t_{h}\right)$ for a $5 \mu \mathrm{L}$ sample

TABLE I

RELATION BETWEEN THE SPEED OF SPIN AND THE TIME OF TRAVELING THROUGH THE MICRO-CHANNEL OF BIO-SAMPLES

\begin{tabular}{cc}
\hline \hline Speed of spin (rpm) & Traveling-time (s) \\
\hline 100 & 5477 \\
200 & 1369 \\
300 & 609 \\
400 & 342 \\
500 & 219 \\
600 & 152 \\
700 & 111 \\
800 & 86 \\
900 & 67 \\
1000 & 55 \\
1100 & 45 \\
1200 & 38 \\
\hline \hline
\end{tabular}

flowing through the micro-channel at different centrifugation speeds using equation (14), which are shown in Table I.

\section{EXPERIMENTS AND RESULTS}

In this section, we present the results of experiments performed to evaluate the theoretical analysis in the previous section. The experiments were performed to measure the spin time and speed for effective passage through the microchannel. Since the accuracy of the volumes delivered and traveled directly affects the experimental results, we first describe approaches which we use to deliver and measure the volume of the bio-samples.

\section{A. Delivery accuracy and measurement of micro-liter volumes of highly viscous bio-samples}

To test the effectiveness of the approach, we used honey as a test biomaterial for convenience since the cost of honey is low while its viscosity is as high as $3000 \mathrm{cP}$ [25] which is close to that of the cubic phase used in the membrane protein crystallization studies [8]. The honey was delivered to the micro-chamber by means of a $250 \mu \mathrm{L}$ Hamilton $^{\circledR}$ microsyringe which is equipped with a PB-600 repeating dispenser from Hamilton that repeatedly dispenses samples of constant volume which was set to $5 \mu \mathrm{L}$.

To check the reproducibility of the micro-syringe dispenser, dispensed volumes were measured by means of an image system as reported in [8]. Briefly, the bio-samples are first dispensed on a glass plate, and then the plate is covered with another glass plate. The dispensed bio-sample is thus sandwiched between the glass slides. A $0.215 \mathrm{~mm}$-thick plate spacer is placed between two glass plates such that the honey sample layer is uniformly thick between the plates. The volume is measured using an imaging system which was developed in our lab based on the toolbox in MatLab ${ }^{\circledR}$ : image processing. The automated image analysis determines the area occupied by the delivered solution, and the volume of delivery is calculated as the product of area and plate spacer thickness.

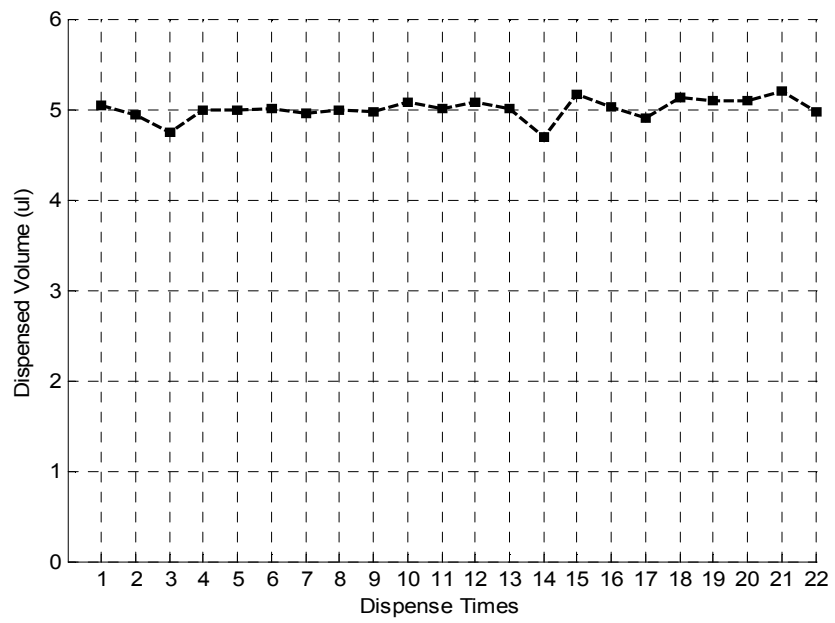

Fig. 6. Accuracy of delivering $5 \mu \mathrm{L}$. 
The accuracy is calculated by measuring the dispensed volumes of 22 trails which is shown in Fig. 6. The results indicate that the deviation for delivering $5 \mu \mathrm{L}$ is less than $1.8 \%$ which is sufficiently accurate for evaluating the performance of the next experiment.

\section{B. Flow control of highly viscous bio-sample}

An Eppendorf $f^{\circledR} 5702$ centrifuge with a Plate Rotor A-438 whose radius is $0.12 \mathrm{~m}$ was used to perform the flow control studies. During the experiment, the $5 \mu \mathrm{L}$ sample is delivered into one chamber of the micro-capsule, and the other chamber is left open with a movable glass slide (see Fig. 7 for further explanation). Under the centrifugal force, the sample travels through the micro-channel to the second chamber onto the glass slide. The method described earlier can be used to measure the volume on the glass.

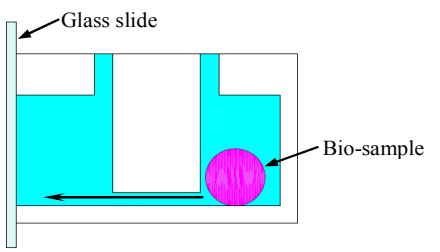

(a)

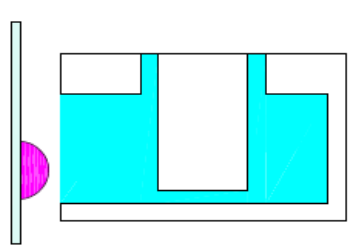

(b)
Fig. 7. Bio-sample travels to the moveable glass slide: (a) a movable glass slide covered at the open chamber (b) bio-sample sticked on the glass slide through micro-channel under the centrifugal force.

Fig. 8 shows the actual volume of the samples that passed through the micro-channel in a period of 60s at different spin speeds. As expected the volume increases with speed. However, the volume recorded levels off at $90 \%$ of the expected volume at the speed of $1,000 \mathrm{rpm}$, i.e., about $10 \%$ cannot be transferred. This is because a thin layer of the viscous bio-sample sticks to the wall of the micro-chamber (recall that the velocity of the bio-sample is zero on the wall of the micro-channel as shown in Figs. 4 and 5), and is unable to flow through the micro-channel. How thin the layer is depends on the time and speed of spin which will be further explained in the next experiment.

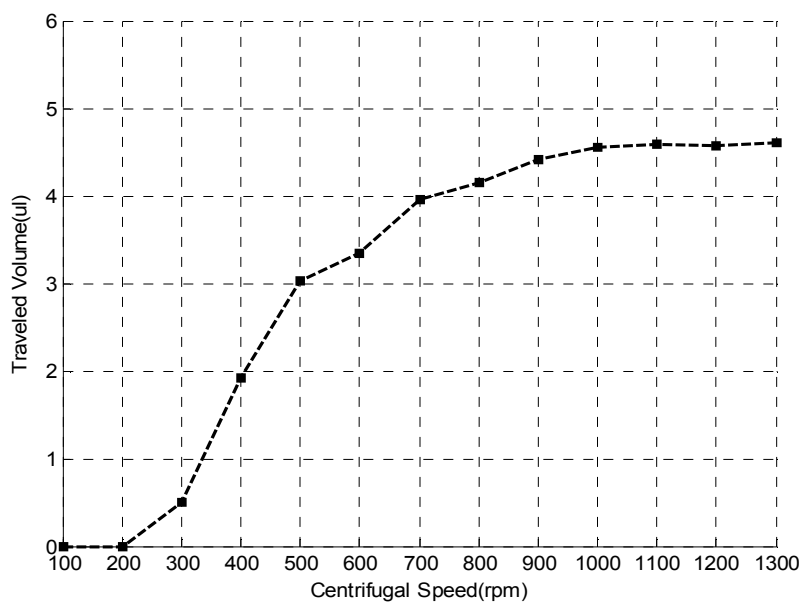

Fig. 8. Transferred volumes under different speeds of spin with a fixed time duration $(60 \mathrm{~s})$.
Next we compare the time-course of passage through the channel at two different speeds, 500rpm and 800rpm. The result is shown in Fig. 9. It is apparent that the transferred volume increases with time and speed. At 800rpm the transferred volume almost reaches $5 \mu \mathrm{L}$ at $150 \mathrm{~s}$. This result shows that the spin time is more important than the speed to completely transfer $5 \mu \mathrm{L}$. However, for $500 \mathrm{rpm}$, even if the spin time is much longer than $150 \mathrm{~s}$, the transferred volume is never close to $5 \mu \mathrm{L}$. We consider two reasons for the above two results. Firstly, the speed of the sample which is close to the wall is very low regardless of the magnitude of the centrifugal force. Thus, it takes extra time for that portion of the sample to flow through the channel. Secondly, a small centrifugal force cannot completely overcome the resistance of the surface to the flow. As a result, a thin layer of the biosample is stuck to the surface and never flows through the channel. This result proves that the spin speed must be greater than certain threshold for effective flow through the micro-channel.

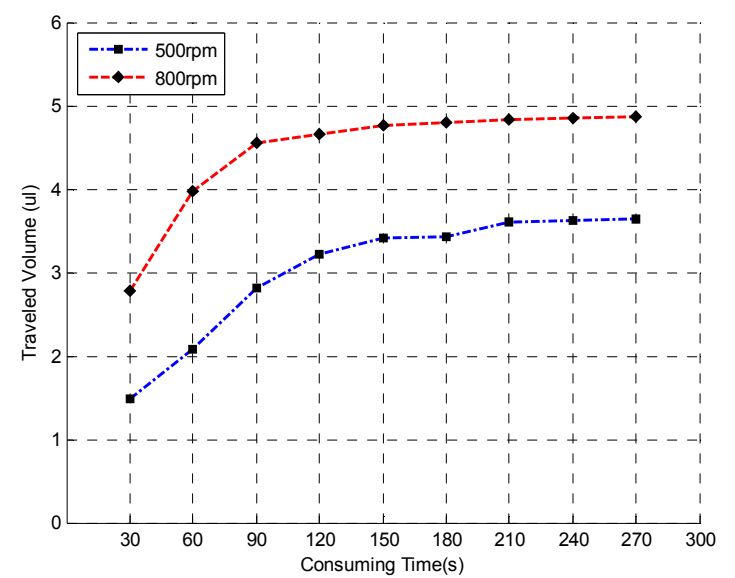

Fig. 9. Relationship between the travelled volume and the time of spin under the speed of $n=500,800 \mathrm{rpm}$, respectively.

The theoretical results using the formulation derived in the previous section are shown in Table I which is for $5 \mu \mathrm{L}$ biosample of $3000 \mathrm{cP}$ to flow through the micro-channel. It is shown when $n=800 \mathrm{rpm}$, the calculated spin time is $86 \mathrm{~s}$, while the actual time approaches 90 s as shown in Fig. 9. The reason for this small discrepancy is because we use the average velocity of flow for the sample as shown in equation (13). In reality, however, it takes much longer time for the thin layer near the wall to flow through (and some never does). This also explains why the volume transferred increases rapidly within 90 s of spin but slowly afterwards. From the above results we conclude that $800 \mathrm{rpm}$ plus $90 \mathrm{~s}$ is an optimal combination for the flow of honey in the microchannel.

\section{CONCLUSIONS}

In this paper, we have presented a mechanism for automatic mixing of highly-viscous bio-samples using microchannel and centrifugation. We first present the design of a micro-capsule for holding and mixing the bio-samples in 
which a micro-channel is built. To understand the physical principle of micro-channel mixing, the study has been focused on the flow of viscous materials in the microchannel. A mathematical model which governs the behavior of the flow has been developed, from which the relationship between the flow speed of the material and the centrifugal force is derived. Furthermore, the relationship between the transferred volume and the required time of spin is developed. Experimental results have shown that the theoretical analysis performed in this paper is fundamental valid although small discrepancy exists which is caused by a thin layer of the sample stuck to the wall of the microchannel and never transferred. The thickness of the layer has a complicated relationship with the centrifugal force and the time of spin, that deserves a careful study in the future.

The results of this paper will facilitate the design of an effective and efficient centrifugal system for high-throughput mixing of highly viscous bio-samples.

\section{REFERENCES}

[1] M. Caffrey, "Membrane protein crystallization," J. Struct. Biol., 142, 2003, pp. 108-132.

[2] V. Cherezov and M. Caffrey, "A simple and inexpensive nanolitervolume dispenser for highly viscous materials used in membrane protein crystallization," J. Appl. Cryst. 38, 2005, pp. 398-400.

[3] E. Landau and J. Rosenbusch, "Lipidic cubic phases - A novel concept for the crystallization of membrane proteins," in Proc. National Academy of Science, 1996, pp. 14,532-14,535.

[4] Luzzati, "X-ray diffraction studies of lipid-water systems," Biological Membranes, Physical Facts and Functions, Vol. 1, Edited by D. Chapman, New York: Academic Press, 1968, pp. 71-123.

[5] Michette and S. Pfuntsch eds. X-rays: the First Hundred Years, John Wiley \& Sons, 1996.

[6] M. Caffrey, "X-ray diffraction as a technique studying the mesomorphic phase properties of lipids," Membranes, Metabolism and Dry Organisms, Edited by A. Leopold, New York: Cornell University Press, 1986, pp. 350-357.

[7] V. Cherezov, A. Peddi, L. Muthusubramaniam, Y. F. Zheng and M. Caffrey, "A robotic system for crystallizing membrane and soluble proteins in lipidic meso phases," Acta. Cryst. D, 60, 2004, pp. 1,7951,807 .

[8] A. Peddi, L. Muthusubramaniam, L., Y.F. Zheng, V. Cherezov, and M. Caffrey, "High-throughput automated system for crystallizing membrane proteins in lipidic mesophases," IEEE Trans. on Automation Science and Engineering, Vol. 4, No. 2, April 2007, pp. 129-140.

[9] W. Chen, A. Peddi, Y.F. Zheng, and M. Caffrey, "Automating crystal harvesting and mounting for high-throughput macromolecular crystallography," in Proc. the 5th World Congress on Intelligent Control and Automation, 2004, China, pp. 4,650-4,655.

[10] A. Peddi, Y.F. Zheng, V. Cherezov, and M. Caffrey, "Automating the dispense of viscous biomaterials," in Proc. 2004 IEEE/RSJ International Conference on Intelligent Robots and Systems, September 28 - October 2, 2004, pp.1,282-1,286.

[11] A. Peddi, Y.F. Zheng, V. Cherezov, and M. Caffrey, "Efficient and effective path for automated dispensing of bio-precipitant solution," in Proc. IEEE Conference on Automation Science and Engineering, August 1-2, 2005, Edmonton, Canada, pp. 61-66.

[12] M. Caffrey, Private Communication, September, 2005.

[13] V. Cherezov, M. Caffrey, "Picoliter-scale crystallization of membrane proteins," J. Appl. Cryst., Vol. 39, 2006, pp. 604-609.

[14] V. Cherezov, E. Yamashita, W. Liu, M. Zhalnina, W. A. Cramer, M. Caffrey, "In meso structure of the cobalamin transporter, BtuB, at 1.95 Å resolution,” J. Mol. Biol., Vol., 364, 2006, pp. 716-734.
[15] S.R. Lamping, H. Zhang, B. Allen, and P.A. Shamlou, "Design of a prototype miniature bioreactor for high throughput automated bioprocessing," Chemical Engineering Science, Vol. 58, 2003, pp. 747-758.

[16] W. A. Duetz, B. Witholt, "Effectiveness of orbital shaking for the aeration of suspended cultures in square-deep well microliter plates," Biochemical Engineering Journal, Vol. 7, 2001, pp. 113-115.

[17] I. Imahdi, F. Baganz, K. Dixon, T. Harrop, D. Sugden and G. J. Lye, " $\mathrm{pH}$ control in microwell fermentations of S. erythraea CA340: influence on biomass growth kinetics and erythromycin biosynthesis," Biochemical Engineering Journal, Vol. 16, 2003, pp. 299-310.

[18] A. Kawai, T., Futami, H. Kiriya, K. Katayama, and K. Nishiawa, "Massproduction system of nearly monodisperse diameter gel particles using droplet formation in a microchannel," in Baba, Y., et al. (Eds.), Micro Total Analysis Systems, Vol. 1, 2002, Kluwer Academic Publishers, the Netherlands, pp. 368-370.

[19] T. Thorson, R. W. Roberts, F. H. Arnold, and S. R. Quake, "Dynamic pattern formation in a vesicle-generating microfluidic device," Physical Review Letters, Vol., 86 (18), 2001, pp. 4,163-4,166.

[20] K. Mae, T. Maki, I. Hasegawa, U. Eto, Y. Mizutani and N. Honda, "Development of a new micromixer based on split/recombination for mass production and its application to soap free emulsifier," Chemical Engineering Journal, Vol. 101, 2004, pp. 31-38.

[21] M. Micheletti, T. Barrett, S. D. Doig, F. Baganz, M. S. Levy and G. J. Lye, "Fluid mixing in shaken bioreactors: implications for scale-up predictions from microlitre-scale microbial and mammalian cell cultures," Chemical Engineering Science, Vol. 61, 2006, pp. 2,9392,949 .

[22] D. Bothe, C. Stemich, and H.-J. Warnecke, "Fluid mixing in a Tshaped micro-mixer," Chemical Engineering Science, 61, 2006, pp. 2,950-2,958.

[23] H. Barnes, J. Hutton, and K. Walters, An Introduction to Rheology, Elsvier, 1989.

[24] K. Sherwin, Thermofluids, New York: Chapman \& Hall, 1996.

[25] http://www.sheepscotmachine.com/viscosity.html. 